University of Nebraska - Lincoln

DigitalCommons@University of Nebraska - Lincoln

2-1-1997

\title{
Monte Carlo simulation of the solid to superliquid phase transition of Langmuir monolayers. II. Characteristics of phase transition
}

\author{
M.D. Gibson \\ University of Nebraska - Lincoln \\ D.R. Swanson \\ University of Nebraska - Lincoln \\ Craig J. Eckhardt \\ University of Nebraska - Lincoln, ceckhardt1@unl.edu \\ Xiao Cheng Zeng \\ University of Nebraska - Lincoln, xzeng1@unl.edu
}

Follow this and additional works at: https://digitalcommons.unl.edu/chemistryeckhardt

Part of the Chemistry Commons

Gibson, M.D.; Swanson, D.R.; Eckhardt, Craig J.; and Zeng, Xiao Cheng, "Monte Carlo simulation of the solid to superliquid phase transition of Langmuir monolayers. II. Characteristics of phase transition" (1997). Craig J. Eckhardt Publications. 10.

https://digitalcommons.unl.edu/chemistryeckhardt/10

This Article is brought to you for free and open access by the Published Research - Department of Chemistry at DigitalCommons@University of Nebraska - Lincoln. It has been accepted for inclusion in Craig J. Eckhardt Publications by an authorized administrator of DigitalCommons@University of Nebraska - Lincoln. 


\title{
Monte Carlo simulation of the solid to superliquid phase transition of Langmuir monolayers. II. Characteristics of phase transition
}

\author{
M. D. Gibson, D. R. Swanson, C. J. Eckhardt, and X. C. Zeng \\ Department of Chemistry and Center for Materials Research and Analysis, University of Nebraska-Lincoln, \\ Lincoln, Nebraska 68588
}

(Received 9 February 1996; accepted 29 October 1996)

\begin{abstract}
The restricted-to-free rotator phase transition of fatty acid monolayers has been modeled using a potential which represents the amphiphiles as planar cross sections of fourfold symmetry. Using much larger system sizes than paper I [D. R. Swanson, R. J. Hardy, and C. J. Eckhardt, J. Chem. Phys. 99, 8194 (1993)], Monte Carlo simulations of the isobaric-isothermal ensemble of model systems with varied number of objects were undertaken to study the effect of system size on the characteristics and order of the phase transition. A peak in the specific heat vs temperature curve was observed near the transition. For each system size, the maxima of the peak showed a linear dependence on the area, which is characteristic of a first order transition. The latter is further confirmed from the observation of a small "van der Waals" loop by increasing the volume at a constant temperature. The result of this simulation matches the qualitative behavior of experimental observations of monolayers, which undergo a weakly first order transition. Additional similarity with experiment was found by the determination that the simulated superfluid phase has the short range translational order and quasilong range bond orientational order characteristic of a hexatic phase. (C) 1997 American Institute of Physics. [S0021-9606(97)52605-5]
\end{abstract}

\section{INTRODUCTION}

Langmuir monolayers are films formed by spreading molecules, such as fatty acids, on an aqueous surface. The component molecules of the monolayer, called amphiphiles because they have hydrophobic and hydrophilic parts, may be ordered conformationally, orientationally, and translationally by applying a surface pressure, $\pi$, at a low temperature. Research in the phase transitions of these films has possible future applications such as molecular switches, and Langmuir monolayers are useful models of biological membranes. ${ }^{1}$ The phase diagram of fatty acid films (Fig. 1) has been well studied, ${ }^{2,3}$ and the high pressure, untilted phases have been consistently observed. The crystalline solid (CS), solid (S), and superliquid (LS) phases are analogous to 3D paraffin crystals. ${ }^{4}$ The lowest temperature phase, the CS phase, exhibits centered rectangular packing of herringbone order, with long range translational order. At higher temperature, the $\mathrm{S}$ phase also has herringbone order and centered rectangular packing, but with more orientational disorder and slightly different intermolecular spacings. The $S$ phase is unable to rotate freely, although restricted rotation is possible (see Fig. 1). At even higher temperature, in the LS phase, the amphiphiles are free to rotate, and packing occurs with hexagonal symmetry. When the discovery of the S to LS transition was first reported by Harkins and Copeland, ${ }^{5}$ surface pressure-area isotherms indicated that the transition was most likely second order. More recent observations by Lundquist $^{6}$ and others ${ }^{3,7}$ have shown that the transition is in fact weakly first order.

The solid (S) to superliquid (LS) phase transition has been simulated in paper I using the Monte Carlo method in the isobaric-isothermal ensemble with a cross-section potential model, ${ }^{8}$ which treats the amphiphiles as planar objects of fourfold symmetry. The true symmetry of amphiphilic cross sections is twofold, but the difference between the long and short axes is assumed to be of negligible importance for the phases studied. The model system undergoes a bound-to-free rotator transition between a solid phase with centered rectangular packing and a liquidlike phase with hexagonal symmetry. These findings are consistent with the experimental results of the S to LS transition. ${ }^{9}$

The model transition appeared to be continuous in the earlier simulation, but no detailed study was done to determine the order of the phase transition. To do this, it is necessary to simulate larger systems (more amphiphiles) and to gather more data near the transition. Because of large fluctuations in the calculation of properties near the transition, more Monte Carlo steps are needed to obtain reliable statistical averages.

In order to distinguish between weakly first order and continuous behavior, a study of the finite-size effect is necessary. For an infinitely large system, first order transitions are discontinuous in internal energy which causes $\delta$-functions in the specific heat at the transition. For finitesize systems, the discontinuities do not occur, and the specific heat vs temperature curve has a finite height and width. For first order transitions, the specific heat maxima are proportional to the volume $L^{d}$ in $d$ dimensions, and the $\delta$-function limit is approached since the width decreases as $L^{-\mathrm{d}}$.

On the other hand, for second order transitions the specific heat is a $\lambda$-function for infinitely large systems and is proportional to $\tau^{-\alpha}$, where $\tau=\left(T-T_{c}\right) / T_{c}, \alpha$ is a critical exponent, and $T_{c}$ is the critical temperature. The specific heat function is broadened for smaller systems. This rounding is due to the correlation length $\xi$ being limited by the system 


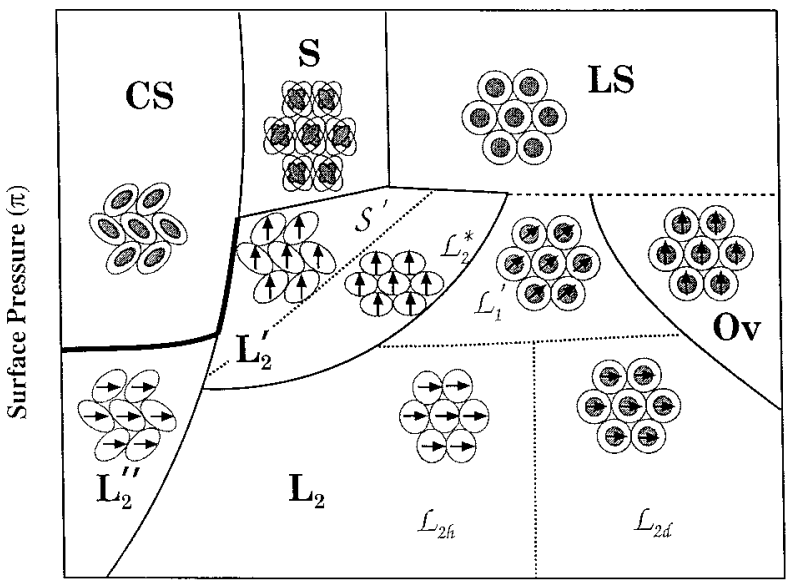

Temperature (T)

FIG. 1. Composite $\pi-T$ phase diagram for fatty acids. Arrows denote direction of tilt, shaded circles denote background disorder, and ellipticity represents the degree of backbone orientational order.

size, and scaling theory ${ }^{10,11}$ predicts the specific heat maxima to grow as $L^{\alpha / \nu}$ and the width to decrease as $L^{-1 / \nu}$, where $\nu$ is related to the correlation length by $\xi \propto \tau^{-\nu}$. The temperature where the maximum occurs also has a size dependence. This temperature shift is described by $\tau=\left[T-T_{c}(\infty)\right] / T_{c}$, where $T$ is the temperature at the maximum specific heat, and $T_{c}(\infty)$ is the critical temperature in the limit of infinite size. The size dependence of the temperature shift is $\tau \propto L^{-1 / \nu}{ }^{10}$ It is important to note that the scaling theory predictions are true only as the limit of infinite size is approached.

The experimental values of the critical exponents have been observed to be distinctly similar for different materials. ${ }^{12}$ This is due to the large fluctuations at the critical point, which makes the molecular details less important. Because of this apparent universality, it is expected that $\alpha$ and $\nu$ should be approximately the same for any $2 \mathrm{D}$ system. The critical exponents for the 2D Ising model have been calculated to be $\alpha=0$, and $\nu=1$. $^{13}$ Assuming similar values hold for the $2 \mathrm{D}$ system studied in this work, the effect of lattice size on the phase transition can be further clarified. If the transition is second order, the specific heat maxima should be constant (proportional to $L^{\alpha / \nu}=L^{0}$ ), and the width and temperature shift should be proportional to $1 / L$. In other words, the maxima should be proportional to the area if the transition is first order, and independent of the system size if the transition is second order.

This simulation is also of interest due to its relation to the study of melting in two dimensions. Much work has been undertaken recently in this area to elicit the nature of the phase transition. A two-dimensional melting transition was first observed in 1962 by Alder and Wainwright in a computer simulation of hard disks. ${ }^{14}$ From their observation of a "van der Waals" loop in the density-pressure curve, they concluded that the transition was first order. Later, Halperin and Nelson ${ }^{15,16}$ and Young ${ }^{17}$ proposed a theory of defect mediated melting based on the work of Kosterlitz and Thouless, ${ }^{18}$ which suggests that melting in two dimensions occurs by two continuous transitions. The first transition is caused by the unbinding of pairs of dislocations present in the solid phase. The resulting free dislocations disrupt the translational order, but preserve bond orientational order. This intermediate phase between the solid and isotropic liquid is called the hexatic phase and is characterized by short range translational order and quasilong ranged bond orientational order. This phase then changes to the isotropic fluid by a second continuous transition. According to HNY theory, this transition occurs by disclination unbinding. There is still some controversy regarding which melting scenario is realized, even for simulations using the same interaction potential. Early simulations of Lennard-Jones atoms in two dimensions by Frenkel and McTague, ${ }^{19}$ and Tobochnick and Chester $^{20}$ supported the HNY theory and seemed to indicate the presence of an intermediate hexatic phase. Monte Carlo simulations of the Lennard-Jones systems by Udink and Frenkel, ${ }^{21}$ supports the assumption of a disclination unbinding transition, but with a defect-rich hexatic phase not predicted by HNY theory. Most simulation results, however, tend to confirm a single first order melting transition. ${ }^{22-26}$ The possibility has even been suggested that $2 \mathrm{D}$ melting is first order at high pressure, but second order at low pressure. $^{16,19}$ This controversy regarding 2D melting is still unresolved; however, much experimental evidence supports the existence of hexatic phases, and the LS phase of amphiphilic monolayers is commonly believed to be hexatic. $^{3,27-30}$

Most simulations of 2D melting have used circularly symmetrical models such as the Lennard-Jones or hard disk systems. In this paper, the anisotropic factor in the potential energy function adds a rotational degree of freedom, which makes direct comparison to 2D melting studies difficult. However, at sufficiently high temperatures, rapid rotation should cause the anisotropic shape to act effectively circular. In this limit, the simulation model should approach the behavior of the isotropic models (i.e., hard disks and LennardJones) often used to study 2D melting.

\section{MODEL}

The packing behavior of a monolayer strongly depends on the cross-sectional shape of each amphiphile, and the hydrophobic tail group is considered to have the dominant effect. $^{7,31}$ Therefore, in developing the model we assumed the amphiphiles are rigid and normal to the surface so that the cross-sectional shape is constant. A highly idealized model was then developed with two goals in mind; (1) simple; (2) capable of capturing essential physics underlying the specific problem. In summary, the component amphiphiles of a monolayer are modeled as planar objects of fourfold symmetry. ${ }^{32}$ The interactions of these shapes is calculated from an anisotropic pair potential that is based on the Lennard-Jones equation with an angle-dependent factor multiplied by the repulsive term, 


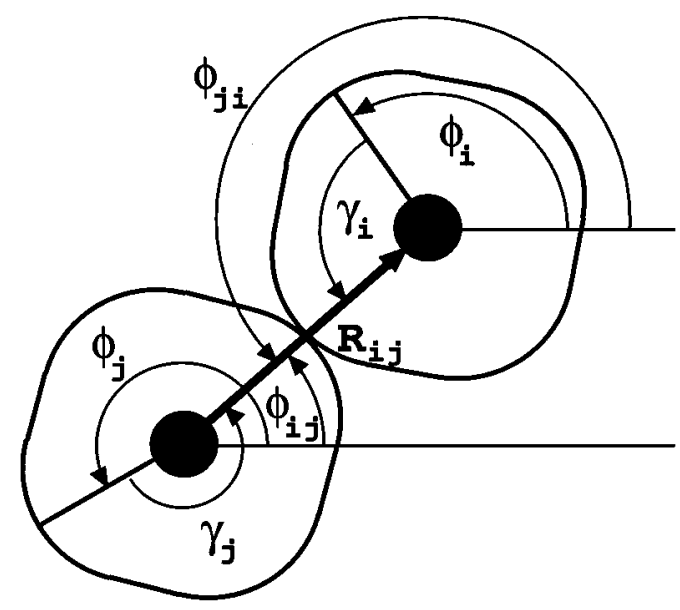

FIG. 2. Model system coordinates.

$$
\begin{aligned}
\phi\left(r_{i j}, \phi_{i j}, \phi_{i}, \phi_{j}\right)= & \epsilon\left(( \frac { \sigma } { r _ { i j } } ) ^ { 1 2 } \left\{1+\frac{1}{10}\left[\cos 4\left(\phi_{i j}-\phi_{i}\right)\right.\right.\right. \\
& \left.\left.\left.+\cos 4\left(\phi_{j i}-\phi_{j}\right)\right]\right\}-\left(\frac{\sigma}{r_{i j}}\right)^{6}\right) .
\end{aligned}
$$

The attractive part of the potential is considered to be isotropic. The coordinates used in this function are shown in Fig. 2. The factor of $\frac{1}{10}$ determines the amount of anisotropy. Since this function evaluates the touching of objects along a line between their centers, it is only accurate for small amounts of anisotropy. The potential was smoothly truncated to zero at $r_{i j}=2.5 \sigma$.

\section{CALCULATIONS AND RESULTS}

Monte Carlo simulations in the isobaric-isothermal ensemble were performed on a Cray J916 supercomputer and a cluster of HP workstations. In all simulations, the reduced surface pressure $\pi^{*}=\pi \sigma^{2} / \epsilon$ is set to be zero. Each particle move consisted of a small random displacement along the $x$ and $y$ direction, and a rotation by a small random angle. After attempting to move all the particles, the boundaries were randomly changed along the $x$ and $y$ axis separately, and the positions of all particles were rescaled without rotating. The contribution to the energy from the pressure was calculated by the product of the surface pressure with the area of the periodic box. Statistical averages based on the isobaric-isothermal ensemble were approximated using the Metropolis algorithm. ${ }^{33}$

In this study, much larger system sizes and longer simulations have been undertaken compared to those reported in paper I. This was necessary to perform the finite size scaling study of the characteristics of the phase transition. System sizes of 144, 196, 256, 400, and 576 particles were used. For each size, a starting configuration of centered rectangular packing with all particle orientational angles aligned was initially run at a low temperature $\left[T^{*}=\left(k_{B} T / \epsilon\right)=0.05\right.$ in Lennard-Jones reduced units; see Fig. 3(a)]. After each run, the temperature was increased by a small amount and the a

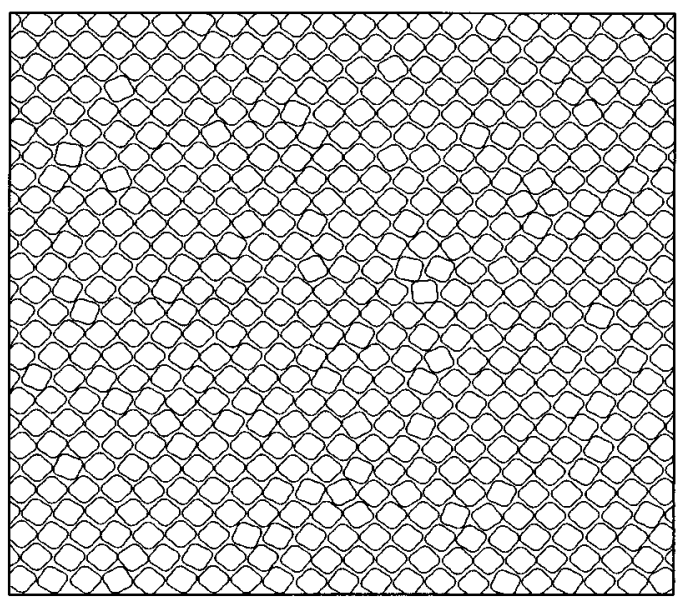

$\mathbf{b}$

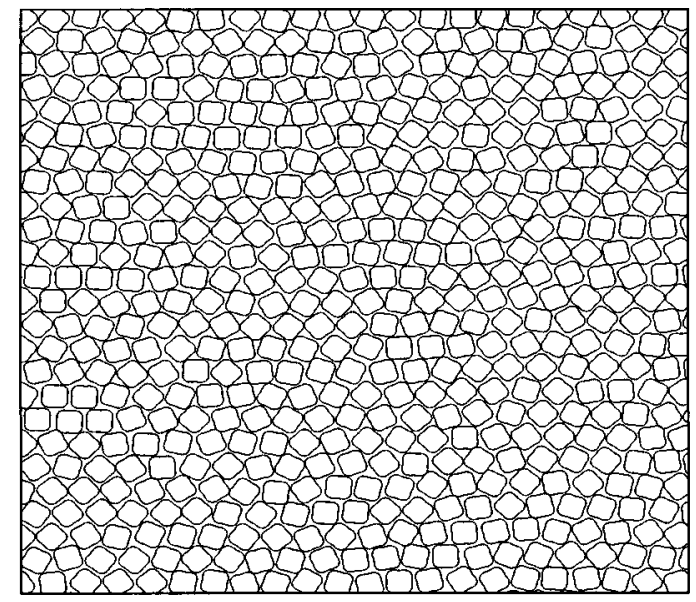

FIG. 3. (a) The monolayer amphiphiles are represented by objects of fourfold symmetry in a plane. In the low temperature $(S)$ phase, there is insufficient thermal energy for free rotation. The molecules pack in a centered rectangular net orientated along the same direction. Each side of the box is set to be $24 \sigma$ initially. (b) In the higher temperature (LS) phase, rotation of the molecules makes them effectively isotropic, and packing occurs in a hexagonal lattice.

final configuration of the preceding run was used as input. This was continued until after the phase transition was observed [Fig. 3(b)]. The potential energy was averaged for each run for at least 250000 attempted moves per particle. The specific heat was calculated from the fluctuation in the potential energy $V$ using the following equation:

$$
C_{\pi}=\frac{\left\langle V^{2}\right\rangle-\langle V\rangle^{2}}{N k_{B} T^{2}}+\frac{3}{2} k_{B}
$$

where $V$ is the total potential energy of the $N$-particle system. 


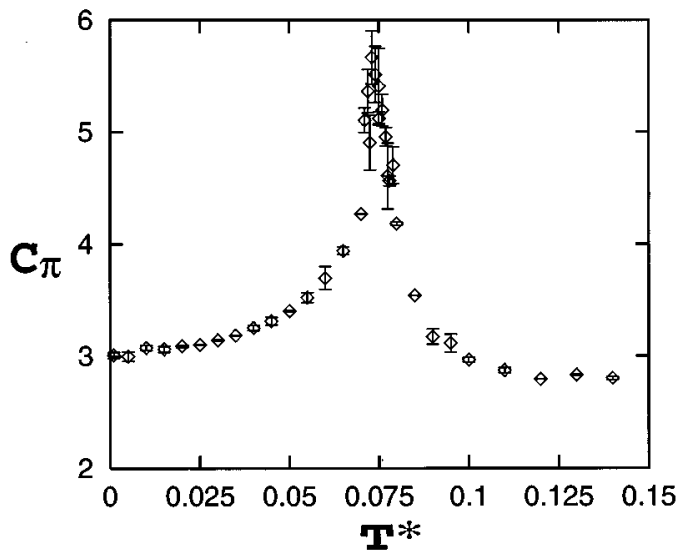

FIG. 4. Specific heat vs temperature in reduced units for $N=100$. The shape of this curve is typical of those used in the finite-size effect study. For these larger sizes, the calculations were more concentrated around the transition temperature.

The specific heat as a function of reduced temperature for 100 particles is shown in Fig. 4. The peak in Fig. 4 demonstrates a characteristic of a phase transition. For a first order transition, the height of the peak should be proportional to the area $\left(L^{2}\right)$, while for a second order transition the height should be independent of the area. To make the finitesize analysis, the average length, $L$, is calculated from the square root of the area of the box. The area is found from the average density. Because of the large statistical fluctuations that occur near the transition temperature, the error bars of the peak widths were very large which render finite-size analysis of peak width inconclusive. This large fluctuation of data contributed also to the error in estimating the temperature of the transition (Fig. 5). On the other hand, the height of the peak can be estimated quite accurately since much less error bars are associated. From Fig. 6, one can see clearly the proportion of peak height to the area, which is characteristic of a first order transition.

To determine if the model system shows hexatic order, the correlation function of the sixfold bond orientational order parameter ${ }^{23}$ was calculated [Fig. 7(a)]. This parameter is defined by the following equation:

$$
g_{6}(r)=\left\langle\psi_{6}(0) \psi_{6}(r)^{*}\right\rangle,
$$

where $\psi_{6}$ is the sixfold bond orientational order parameter,

$$
\psi_{6}(r)=\sum_{k=1}^{6} \exp \left(6 i \theta_{j k}\right),
$$

where $\theta_{j k}$ is the angle of an imaginary bond between nearest neighbors $j$ and $k$ measured against an arbitrary axis, and the summation is over six nearest neighbors of particle $j$. The envelope of the correlation function $g_{6}(r)$ is expected to decay exponentially with increasing system size for an isotropic liquid, or to decay algebraicly for a hexatic phase. The radial distribution function was also calculated to determine the range of the translational order, and its envelope is expected to show an exponential decay for the LS phase [Fig. 7(b)]. These correlation functions were calculated for a large

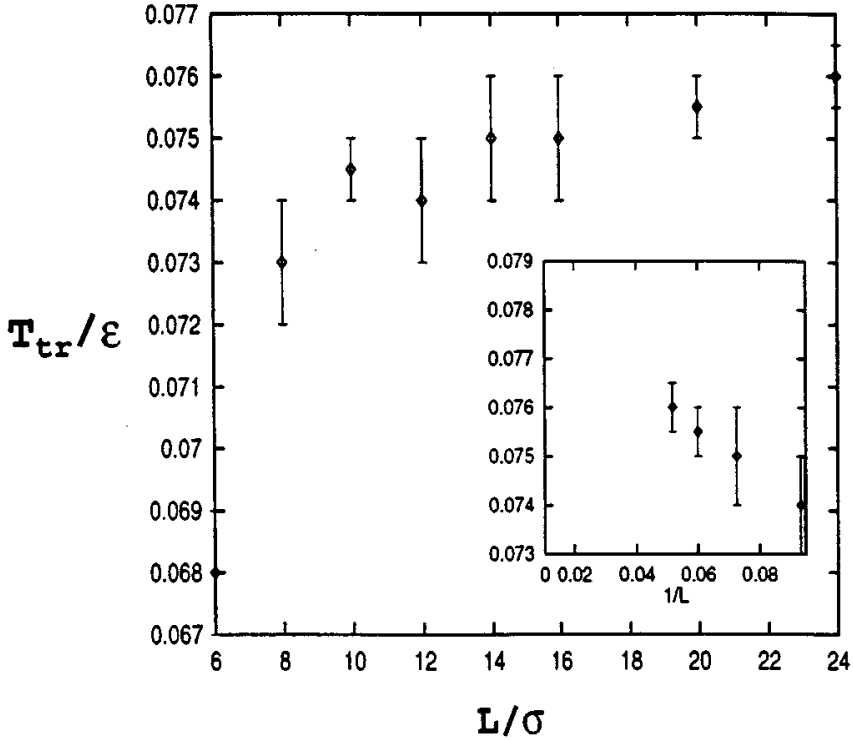

FIG. 5. Transition temperature vs $L$. This shows the increase in the temperature at the specific heat maxima vs $L$. This can be extrapolated to estimate the transition temperature in the thermodynamic limit. The insert shows the transition temperature plotted vs $1 / L$. Extrapolating to zero gives a temperature of $T_{\mathrm{tr}}^{*}(\infty)=0.078$.

system of 4096 particles. Prior to this calculation, the system was equilibrated for 500000 Monte Carlo cycles. To unambiguously assign the nearest neighbors in the $g_{6}(r)$ calculation, the Voronoi construction should be used; however, to reduce the computational effort, a cutoff distance of $1.3 \sigma$ was used to determine the nearest neighbors. This value was determined by examining the location of the first and second peaks in the radial distribution function. The Voronoi con-

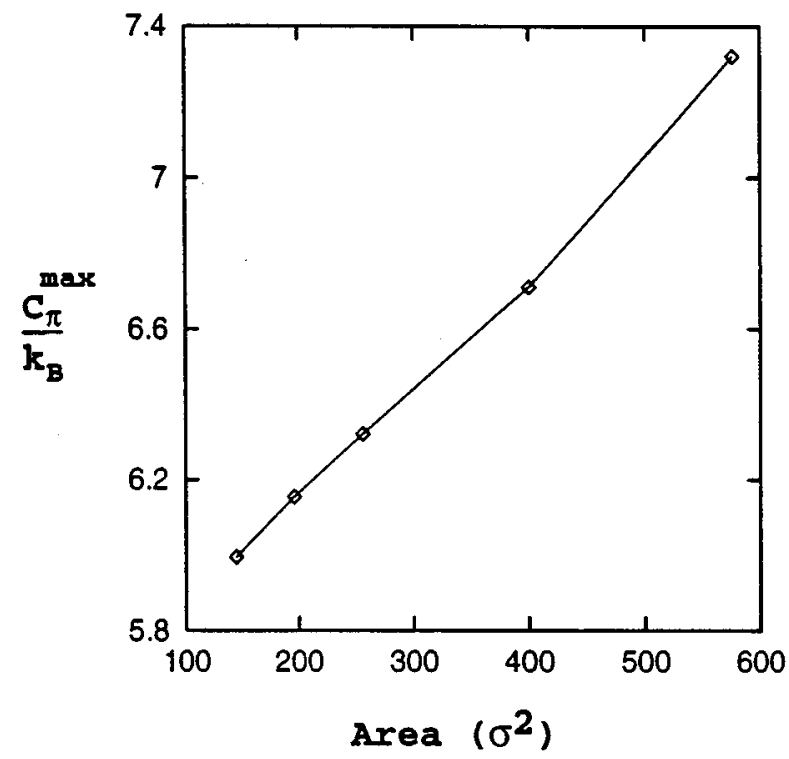

FIG. 6. Specific heat maxima vs $L^{2}$, for $N=144,196,256,400$, and 576. This linear behavior is indicative of a first order transition. 


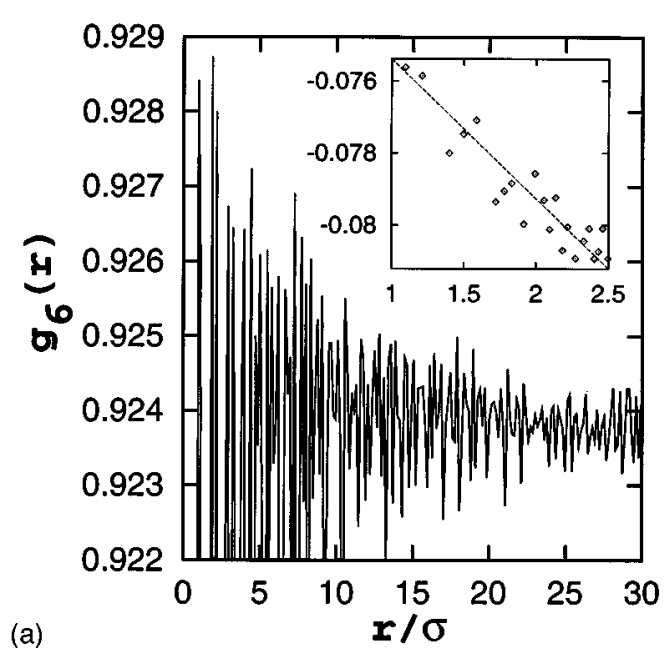

(a)

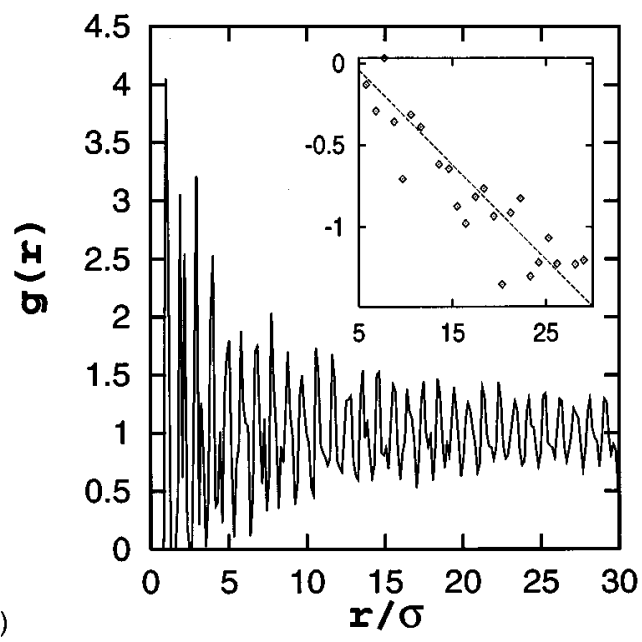

FIG. 7. (a) Correlation function of bond orientational order $g_{6}(r)$ at $T^{*}=0.1$. The insert is the natural logarithm of the $g_{6}(r)$ envelope plotted against the natural logarithm of distance to show $g_{6}(r)$ decays algebraically with increasing $L$ which is indicative of quasilong ranged bond orientational order. The algebraic exponent $\eta_{6}$ can be estimated from this slope to be approximately 0.004. (b) Correlation function of translational order $g(r)$ at $T^{*}=0.1$. In the insert, the natural logarithm of the envelope of $g(r)-1$ is linear when plotted against $r$ demonstrating the exponential decay characteristic of short range order. From the slope of this line the correlation length $\xi$ can be estimated to be around $17 \sigma$.

struction was used on a single typical configuration to verify that the cutoff method is adequate to find the nearest neighbors.

To further study the phase transition, constant volume Monte Carlo simulations were run for several volumes near the transition where $\pi^{*}$ is close to zero. The temperature was constant for all runs, and the volume was gradually expanded. In the simulations, the aspect ratio of the box was changed to allow the centered rectangular lattice to become hexagonal. The reduced surface pressure was calculated as a function of reduced density $\left(\rho^{*}=\rho \sigma^{2}\right)$ and showed the presence of a small "van der Waals" loop (Fig. 8).

\section{DISCUSSION AND CONCLUSION}

A bound-to-free rotator transition was observed in these simulations that is qualitatively similar to the restricted-to-

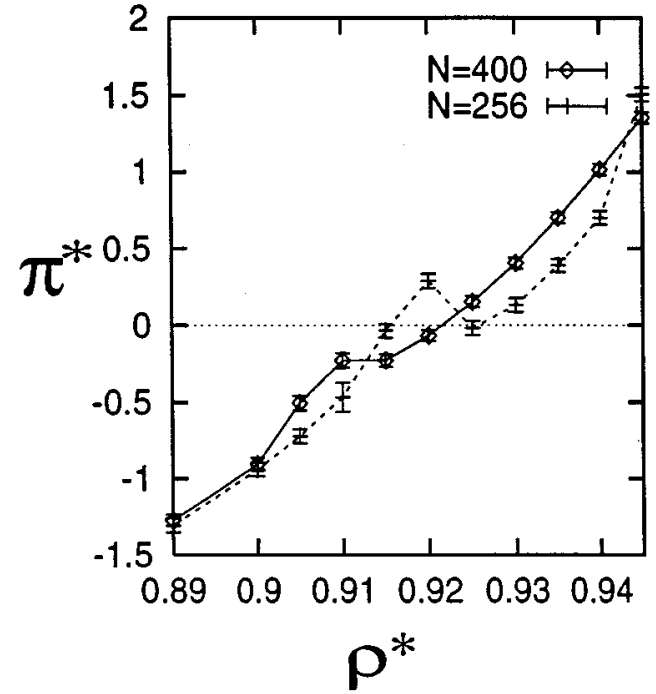

FIG. 8. $\Pi^{*}-\rho^{*}$ curve from NVT ensemble Monte Carlo calculation at $T^{*}=0.075$ for two system sizes. The observed van der Waals loops become smaller with increasing size.

free rotator ( $\mathrm{S}$ to $\mathrm{LS}$ ) transition experimentally observed for fatty acid monolayers. To determine the order of this transition, the specific heat maxima were calculated for system sizes of $L=12,14,16,20$, and 24 . The specific heat maxima clearly show the linear dependence on $L^{2}$ that is expected for a first order transition (Fig. 6). Additional confirmation of the first order character of the transition comes from the observation of a small "van der Waals" loop shown in Fig. 8. This loop is shown for two system sizes and it can be seen that the loop becomes smaller as the size is increased. This is expected of a first order transition. The shift in the loops to higher pressure and density probably is a result of finite size effects.

The anisotropy in the cross sections allows a rotational degree of freedom. At very low temperature, there is insufficient energy to overcome the barrier to rotation, and the amphiphiles pack in a lattice of minimum energy with the rotational angles fixed and aligned. With a cross section of fourfold symmetry, this lattice is centered rectangular. At sufficiently high temperature, rotation makes the cross section effectively isotropic since the rotation occurs freely, and therefore the packing occurs in a hexagonal lattice. It is important to emphasize that this does not mean the superliquid phase is isotropic, as it may retain bond orientational order.

The radial distribution function is predicted to decay exponentially at large distance i.e., $g(r)-1 \sim e^{-r / \xi}$ where $\xi$ is the correlation length. Two dimensional melting theory also predicts for hexatic phases, algebraic decay of the bond orientational correlation function $g_{6}(r) \sim r^{-r_{6}}$, where $\eta_{6}$ is temperature dependent. ${ }^{34}$ The bond orientational correlation function $g_{6}(r)$ is shown for $T^{*}=0.1$ and $N=4096$ [Fig. $7(\mathrm{a})]$. The envelope of $g_{6}(r)-1$ shows an algebraic decay which indicates quasilong range bond orientational order characteristic of a hexatic phase. The translational order is found to be short range since the envelope of the radial distribution function shows a much faster exponential decay 
[Fig. 7(b)]. This seems to match well with the experimentally observed LS phase which is commonly considered to be hexatic. However, the "hexatic" phase seen in the simulations is not the same as the intermediate phase predicted from the HNY theory of 2D melting. The appearance of a hexatic phase is explained in two-dimensional melting theory by the unbinding of dislocation pairs to form free dislocations in the lattice. Examination of the configurations produced by the Monte Carlo simulations does not show the presence of dislocations. This was confirmed by using the Voronoi construction which gave a coordination number of six for all the particles. No continuous transition from a solid to hexatic phase was observed at the temperatures studied, nor was a continuous transition from a hexatic to isotropic liquid seen. These transitions are predicted by the theory of defect mediated melting, and may occur at temperatures higher than those simulated here. The observed hexaticlike phase is therefore not caused by free dislocations in the lattice as in HNY theory, but possibly from the anisotropy of the cross sections. Also, the bond orientational order decays extremely slowly and almost appears to be constant. This behavior is not seen in experimental hexatic phases and may be an artifact of the simulation, perhaps caused by the periodic boundary conditions. Another possible explanation comes from the very slow equilibration of bond orientational order in large systems. It may be that the long-range order results from insufficient equilibration to remove the order present in the initial lattice. More study needs to be done to determine conclusively the presence or absence of hexatic order in the high temperature phase.

This phase transition behavior is expected to be similar for any anisotropic cross-sectional shape model system. The high temperature (LS) phase should still be approximately hexagonal since it occurs when the anisotropy is averaged out due to the rotation. Different cross-sectional shapes may cause a change in the low temperature packing, as well as the temperature of the transition. For example, the true cross section of simple fatty-acid amphiphiles is slightly rectangular in shape. This deviation from fourfold symmetry would have an effect on the packing of the S phase and the transition temperature. With twofold symmetry, the amphiphiles could pack with herringbone order, as is common for alkanes, and is the most likely structure of the S and CS phases. Further improvement of the model would be needed in order to accurately simulate various structures of the low temperature phases (e.g., CS to S transition) of Langmuir monolayers (see Fig. 1); for example, a much more realistic model for 3D long-chain amphiphilic molecules is nonrigid bond full-atom model. ${ }^{35}$

In conclusion, a bound-to-free rotator transition is observed in isobaric-isothermal Monte Carlo simulations of a highly idealized 2D cross-section potential model, which matches the qualitative behavior observed by experiment. The transition has been found to be weakly first order, and the presence of a possibly hexatic phase corresponding to the LS phase has been observed. This result supports the premise of Langmuir, ${ }^{30}$ that the packing of monolayers is determined predominantly by the cross-sectional shape of the constituent amphiphiles. This study, therefore, demonstrates the usefulness of simple idealized models based on cross-sectional shape to reveal essential physical behavior of the untilted phases of amphiphilic monolayers.

\section{ACKNOWLEDGMENTS}

This work was supported by the National Science Foundation, Office of Naval Research, and by the Research Council of the University of Nebraska-Lincoln. Acknowledgment is also made to the donors of the Petroleum Research Fund administered by the American Chemical Society for partial support of this work.

${ }^{1}$ G. L. Gaines, Jr., Insoluble Monolayers at Liquid-Gas Interface (Interscience, New York, 1966).

${ }^{2}$ I. R. Peterson, V. Brzezinski, and R. M. Kenn, Langmuir 8, 2995 (1992).

${ }^{3}$ A. M. Bibo, C. M. Knobler, and I. R. Peterson, J. Phys. Chem. 95, 5591 (1991)

${ }^{4}$ J. Doucet, I. Denicolo, and A. Craievich, J. Chem. Phys. 75, 1523 (1981); I. Denicolo, J. Doucet, and A. F. Craievich, ibid. 78, 1465 (1983); E. B. Sirota, H. E. King, Jr., D. M. Singer, and H. H. Shao, ibid. 98, 5809 (1993)

${ }^{5}$ W. D. Harkins and L. E. Copeland, J. Chem. Phys. 10, 272 (1942).

${ }^{6}$ M. Lundquist, Chem. Scr. 1, 5 (1971).

${ }^{7}$ M. C. Shih, T. M. Bohanon, J. M. Mikrut, P. Zshack, and P. Dutta, Phys. Rev. A 45, 5734 (1992)

${ }^{8}$ D. R. Swanson, R. J. Hardy, and C. J. Eckhardt, J. Phys. Chem. 99, 8194 (1993).

${ }^{9}$ C. M. Knobler and R. C. Desai, Annu. Rev. Phys. Chem. 43, 207 (1992).

${ }^{10}$ M. S. Challa, D. P. Landau, and K. Binder, Phys. Rev. B 34, 1841 (1986).

${ }^{11}$ M. E. Fisher, in Critical Phenomena, edited by M. S. Green (Academic, New York, 1971).

${ }^{12}$ M. E. Fisher, in Critical Phenomena, edited by F. J. W. Hahne (Springer, New York, 1983).

${ }^{13}$ H. E. Stanley, Introduction to Phase Transitions and Critical Phenomena (Oxford, New York, 1971).

${ }^{14}$ B. J. Alder and T. E. Wainwright, Phys. Rev. 127, 359 (1962).

${ }^{15}$ B. I. Halperin and D. R. Nelson, Phys. Rev. Lett. 41, 121 (1978).

${ }^{16}$ D. R. Nelson and B. I. Halperin, Phys. Rev. B 19, 2457 (1979).

${ }^{17}$ A. P. Young, Phys. Rev. B 19, 1855 (1979).

${ }^{18}$ J. M. Kosterlitz and D. J. Thouless, J. Phys. C 6, 1181 (1973).

${ }^{19}$ D. Frenkel and J. P. McTague, Phys. Rev. Lett. 42, 1632 (1979).

${ }^{20} \mathrm{~J}$. Tobochnick and G. V. Chester, in Proceedings of the International Conference on Ordering in Two Dimensions, edited by S. K. Sinha (North-Holland, New York, 1980), p. 339.

${ }^{21}$ C. Udink and D. Frenkel, Phys. Rev. B 35, 6933 (1987).

${ }^{22}$ F. Abraham, Phys. Rep. 80, 339 (1981).

${ }^{23}$ A. F. Bakker, C. Bruin, and H. J. Hilhorst, Phys. Rev. Lett. 52, 449 (1984)

${ }^{24}$ K. J. Strandburg, J. A. Zollweg, and G. V. Chester, Phys. Rev. B 30, 2755 (1984).

${ }^{25}$ J. Lee and K. J. Strandburg, Phys. Rev. B 46, 11190 (1992).

${ }^{26}$ H. Weber, D. Marx, and K. Binder, Phys. Rev. B 51, 14636 (1995).

${ }^{27}$ T. M. Fischer, R. Bruinsma, and C. M. Knobler, Phys. Rev. E 50, 413 (1994).

${ }^{28}$ J. Ruiz-Garcia, X. Qiu, M. W. Tsao, G. Marshall, C. M. Knobler, G. A. Overbeck, and D. Möbius, J. Phys. Chem. 97, 6955 (1993).

${ }^{29}$ V. M. Kaganer and E. B. Loginov, Phys. Rev. Lett. 71, 2599 (1993).

${ }^{30}$ C. M. Knobler, J. Phys. Condensed Matter 3, S17 (1991).

${ }^{31}$ I. Langmuir, J. Am. Chem. Soc. 39, 1848 (1917).

${ }^{32}$ Objection may be made that the cross section is actually slightly rectangular. Although true, adjustment for this is not worth the significant increase in calculational difficulty since the same physics of the S to LS transition may be expected for the true shape albeit at a different temperature.

${ }^{33}$ N. Metropolis and S. Ulam, J. Am. Stat. Assoc. 44, 335 (1955).

${ }^{34}$ M. P. Allen, D. Frenkel, W. Gignac, and J. P. McTague, J. Chem. Phys. 78, 4206 (1983)

${ }^{35}$ See, for example, J. Hautman and M. L. Klein, J. Chem. Phys. 91, 4994 (1989); S. Shin, N. Collazo, and S. A. Rice, ibid. 96, 1352 (1992). 